\title{
7 EHRENAMT UND BILDUNG FÜR GeFLÜCHTETE IM LÄNDLICHEN RAUM
}

Die vorangegangenen Abschnitte haben Bildung hauptsächlich in Bezug auf den formalen Bildungssektors beschrieben. Dabei wurden Rahmenbedingungen und Charakteristika unterschiedlicher Angebote für geflüchtete Kinder, Jugendliche und Erwachsene (unter anderem die Pflichtschule, Basisbildungs- und Pflichtschulabschlusskurse) in den Fokus genommen. Bildung findet jedoch auch informell statt, wobei Ehrenamtliche hierbei eine bedeutende Rolle einnehmen. Vielerorts wurden parallel mit der Aufnahme von Geflüchteten rund um das Jahr 2015 Unterstützungsinitiativen gegründet, wobei ehrenamtlicher Deutschunterricht häufig eine zentrale Säule darstellte. In diesem Kapitel werden nun einige Charakteristika ehrenamtlicher Bildungsarbeit dargelegt sowie die Bedeutung zivilgesellschaftlichen Engagements im Allgemeinen für die lokale Integration von Geflüchteten im ländlichen Raum beleuchtet.

\subsection{Strukturwandel im Ehrenamt}

Mit der Ankunft von tausenden Menschen, die 2015 aus den Kriegs- und Krisengebieten des Nahen Ostens geflohen waren, begannen sich zahlreiche Bürger/innen spontan für die Geflüchteten zu engagieren. Vielfach wurden diese freiwilligen Tätigkeiten auch als zivilgesellschaftliches oder bürgerschaftliches Engagement bezeichnet ${ }^{27}$. Das rezente ehrenamtliche Engagement für Asylwerber/innen und Asylberechtigte unterscheidet sich vom klassischen Ehrenamt in der Organisationsform und der inhaltlichen Ausgestaltung in vielerlei Hinsicht: War das klassische Ehrenamt noch stärker mit „Amt" und „Ehre“ verbunden, so wird im neuen zivilgesellschaftlichen Engagement der Sinn weniger aus der Organisation selbst, sondern aufgrund der Inhalte und Zielgruppen generiert. Die Termini „bürgerschaftliches“ oder „,zivilgesellschaftliches Engagement“ betonen zudem die Intention der Handlung als Ausdruck politischer Partizipation aus der Identität der Bürgerin beziehungsweise des Bürgers (SCHÜHRER 2019, pp. 13 ff.). Im Mittelpunkt steht hier die praktische Hilfe, also die konkrete Aufgabe sowie die persönliche Beziehung zu den Geflüchteten. Die Organisationsform verändert sich von formellen Verbänden hin zu informellen Zusammenschlüssen und Initiativen. Starre Hierarchien, Regelungen und Ämter mit umfassender Verantwortung werden eher abgelehnt. An deren Stelle tritt das flexible zivilgesellschaftliche Engagement, das in seiner Dauer wesentlich kurzfristiger und projektbezogener ist und welches auch stärker den Anspruch auf Partizipation und Mitsprache erhebt. Das inhaltliche Interesse steht dabei deutlich im Vordergrund und muss zur eigenen Biografie passen (ebd., pp. 18 ff., 155 ff.; BMFSFJ 2017, pp. 121 ff.).

27 In dieser Publikation werden die Begriffe ehrenamtliches, zivilgesellschaftliches und freiwilliges Engagement synonym verwendet. 
Ein Spezifikum dieser Entwicklung war die Mobilisierung der Bevölkerung zur ehrenamtlichen Tätigkeit über die mediale Berichterstattung (KARAKAYALI \& KLEIST 2015, p. 31). Zudem ist der im Vergleich zum „klassischen Ehrenamt“ hohe Frauenanteil bemerkenswert; dieses ist nämlich, im Gegensatz zu vielen Initiativen, die seit 2015 entstanden sind, überwiegend von Männern dominiert (ebd., p. 15). Zivilgesellschaftliches Engagement für Geflüchtete umfasst viele Tätigkeitsbereiche und Handlungsfelder, die zumeist sehr zeitintensiv sind. Eine Studie über die Strukturen und Motive der ehrenamtlichen Flüchtlingsarbeit in Deutschland kam zu dem Ergebnis, dass die Begleitung bei Behördengängen, Sprachunterricht, Übersetzungsarbeit, Sozial- und Integrationsberatung, aber auch Fahrtendienste und Hilfe bei der Wohnungssuche zu wesentlichen Aktionsgebieten der Ehrenamtlichen gehören (ebd., p. 28). Sie verbringen somit viel Zeit mit den Geflüchteten, was wiederum dazu führt, dass sie mitunter enge freundschaftliche Kontakte aufbauen.

\subsection{Zwischen Aktivismus und humanitärer Hilfe: Zum politischen Aspekt ehrenamtlichen Engagements für Geflüchtete}

Im Hinblick darauf, ob zivilgesellschaftliches Engagement für Geflüchtete per se als politisches Statement zu verstehen ist, oder im Bereich der karitativen Hilfe als apolitisch bezeichnet werden kann, sind sich (Sozial-)Wissenschaftler/innen uneins. Auf der einen Seite des Spektrums steht die karitative, praktisch-organisatorische Hilfe beziehungsweise der ,problem solving approach“ (DE JONG \& ATAÇ 2017), auf der anderen das explizite politische Engagement im Bereich der nationalen Flüchtlingspolitik, bei DE JoNG und ATAÇ als „critical approach“ bezeichnet. Einige Wissenschaftler/ innen betonen die fließenden Übergänge zwischen den beiden Bereichen (EvERS \& Klie 2018, p. 520; Cantat \& Feischmidt 2019). Fleischmann und Steinhilper (2017) argumentieren beispielsweise, dass die rezenten Ehrenamtlichen zwar selten politisch-transformative Ziele verfolgen und die praktische Hilfe deutlich im Vordergrund steht, ihr Engagement jedoch nicht als apolitisch charakterisiert werden sollte. Schließlich können Freiwillige im Laufe der Zeit durch den intensiven Kontakt mit den Geflüchteten ihre politischen Überzeugungen überdenken und eine kritischere Haltung gegenüber der staatlichen Flüchtlingspolitik einnehmen. VANDEVOORDT und VERSCHRAEGEN (2019) bezeichnen diese Gleichzeitigkeit des politischen und apolitischen Elements als „subversive humanitarianism“. Zwar sind die Aktivitäten der Ehrenamtlichen vordergründig ohne politische Intention, implizit kann das zivilgesellschaftliche Engagement für Geflüchtete aber auch als Kritik an der nationalen Flüchtlingspolitik verstanden werden. Auch KaraKaYALI (2017, p. 17) erkennt im neuen Ehrenamt für Geflüchtete eine „Dimension politischer Rahmung und Artikulation“. Zivilgesellschaftliches Engagement im Bereich Flucht und Asyl kann sich aber auch gegen die Geflüchteten richten. Beispiele dafür sind die Demonstrationen der PEGIDA- 
Bewegung ${ }^{28}$ in Deutschland oder die Unterschriftenaktion gegen die Unterbringung weiterer Asylwerber/innen in St. Andrä-Wördern. Es sollte daher behutsam mit einer positiv-wertenden Haltung gegenüber politischen Ausdrucksformen des Engagements bei gleichzeitiger Abwertung der praktischen Hilfe umgegangen werden.

Vereinzelt wird humanitäres Engagement als unkritisches Helfen abgetan, das lediglich unterdrückende staatliche Strukturen reproduziere. VAN DYK und MisBaCH (2016, p. 219) sehen den „Überdruss am politischen Geschäft“ der Ehrenamtlichen und die karitative Hilfe im Hinblick darauf als durchaus bedenklich. Sie befürchten langfristig einen Abbau von staatlichen Dienstleistungen und Infrastruktur. Wenngleich KARAKAYALI (2018, p. 14) darauf hinweist, dass es dafür noch keine Belege gibt, merkt er an, dass die systematische Einbindung der Ehrenamtlichen und der Aufbau von Koordinationsstellen auf Landes- und Bundesebene dieser Entwicklung zumindest die strukturelle Basis dafür bieten könnten. Dies stellt eine Lesart des rezenten ehrenamtlichen Engagements für Geflüchtete dar. Zivilgesellschaftliches Engagement für Geflüchtete kann aber auch unter dem Blickwinkel der Stärkung des sozialen Zusammenhalts, der Demokratieförderung und im Hinblick auf gesamtgesellschaftliche Solidarität betrachtet werden. WaGner (2019, p. 227) fasst die unterschiedlichen Perspektiven prägnant zusammen:

„Man kann das freiwillige Helfen in seiner Funktion für das Gemeinwesen analysieren und es entweder als Ausdruck und Katalysator der Aushöhlung des Wohlfahrtsstaates kritisieren [...] oder das dadurch entstehende Sozialkapital aus einer kommunitaristischen Perspektive als essentiell für den gesellschaftlichen Zusammenhalt verteidigen [...]. “

Obwohl die Motive der Ehrenamtlichen im Zuge der Feldforschungen nicht explizit erhoben wurden, ist davon auszugehen, dass sie individuell sehr vielfältig waren. Manche erzählten, dass ihnen die freiwillige Arbeit Freude bereite, andere betteten ihr Engagement in moralisch-religiöse Überzeugungen, in diesem Fall der christlichen Nächstenliebe, ein. Wieder andere betonten, dass es ihnen bei ihrer ehrenamtlichen Tätigkeit auch um ein solidarisches und nachbarschaftliches Miteinander gehe:

„,Also mir geht's um ein gutes Zusammenleben, [...] das ist meine Motivation. Mit Geflüchteten, aber auch überhaupt diese Vielfalt, die es im Ort gibt, als Ressource zu sehen. [Dass es] da ein gutes Zusammenleben gibt und eine Willkommenskultur und nicht so eine grade so aktuelle Ablehnungskultur, wie wir sie von der Politik vorgelebt kriegen. “ (Interview OBMANN VEREIN „GRENZENLOS“ 2018)

Ein spannendes Forschungsfeld, das im erweiterten Sinn auch die Debatte um den politischen Impetus aufgreift, stellt die ehrenamtliche Hilfe im Hinblick auf Rezip-

28 Die „Patriotischen Europäer gegen die Islamisierung des Abendlandes“ veranstalten seit Ende des Jahres 2014 regelmäßig Demonstrationen gegen eine ihrer Meinung nach zu offene Flüchtlingspolitik in Deutschland. Es besteht dabei ein Naheverhältnis zu anderen rechten und rechtsextremen Gruppierungen. Die Teilnehmer/innen der Veranstaltungen fielen immer wieder mit rassistischen und antisemitischen Aussagen auf. 
rozitätserwartungen dar. Laut WAGNER (2019) kann freiwilliges Helfen als Gabe im Mauss'schen Sinn verstanden werden. Gaben stiften Beziehungen, die von Gegenseitigkeit, einseitiger Abhängigkeit oder symbolischer Ungleichheit geprägt sein können - je nachdem, wer welche Gabe wann womit erwidert. Beim wohltätigen Helfen werden Gaben einseitig ausgetauscht, was Ungleichheit erzeugt. Der karitative Ansatz stellt in gewisser Hinsicht eine Ausnahme dar, da sie im Gegensatz zur solidarischen Hilfe keine Gegenleistung voraussetzt. Allerdings ist auch die wohltätige Gabe nicht völlig frei von Reziprozitätserwartungen (ebd., p. 228). In ihrer qualitativen Studie kam WaGNER zu dem Ergebnis, dass Ehrenamtliche im ländlichen Raum keine direkte Reziprozität in Form einer Gegengabe erwarten, sich jedoch häufig Anerkennung durch Dankbarkeit erhoffen. Ebenso möchten Ehrenamtliche im Kontakt mit den Geflüchteten oftmals Einfluss auf deren Überzeugungen und Werte nehmen, zum Beispiel wenn es um Genderrollen geht. Die Wechselseitigkeit des Gabenaustausches findet somit auf einer symbolischen Ebene statt. Viele Ehrenamtliche betten ihr Engagement in einen gesamtgesellschaftlichen Kontext ein und erwarten im Gegenzug das, was man in soziologischen Termini als ,generalisierte Reziprozität“ bezeichnet. Damit ist gemeint, dass Gaben und Leistungen indirekt und/oder zeitverzögert zwischen zwei oder mehreren Parteien ausgetauscht werden (vgl. STEGBaUer 2011, p. 67). Aus dieser Perspektive stehen Geflüchtete gewissermaßen in der Schuld, der Aufnahmegesellschaft oder den Ehrenamtlichen, im Gegenzug für die Unterstützung, etwas zurückzugeben. Dies kann beispielsweise besonderes Engagement bei der Suche nach einem Arbeitsplatz oder das Anstreben anderer Integrationsleistungen umfassen. Sollten diese Reziprozitätserwartungen nicht erfüllt werden, kann es auf beiden Seiten zu Enttäuschungen kommen. Dennoch betont WAGNER (2019), dass generalisierte Reziprozität die symbolische Ungleichheit zwischen Ehrenamtlichen und Geflüchteten mindern kann.

\subsection{Bildung und Ehrenamt}

Bildung besitzt KLEIST zufolge eine doppelte Funktion; sie ist sowohl Instrument als auch Ziel und Kennzeichen von Integration. In beiden Bereichen spielen Ehrenamtliche eine zentrale Rolle. Eine der am häufigsten unentgeltlich erbrachten Leistungen für Geflüchtete stellte der ehrenamtliche Deutschunterricht dar (KLEIST 2017, p. 27). In allen untersuchten Gemeinden gab es zivilgesellschaftliche Initiativen, die sich dem Sprachunterricht verschrieben hatten. Gerade für Asylwerber/innen, die von staatlichen Integrationsmaßnahmen weitgehend ausgeschlossen sind, stellt der ehrenamtliche Deutschkurs eine wertvolle Ressource dar, um sich Grundkenntnisse der Landessprache anzueignen. Sich auf Deutsch in Alltagssituationen beim Einkaufen oder in den öffentlichen Verkehrsmitteln ausdrücken zu können, verhilft nicht nur zu mehr Selbstständigkeit und gibt Sicherheit; durch den Kontakt zu den Ehrenamtlichen entstehen häufig freundschaftliche Beziehungen, die über das Deutschlernen hinausgehen. Dieses soziale Kapital kann wiederum dazu führen, Zugang zu formalen Bildungsangeboten zu 
bekommen, da Ehrenamtliche hierbei häufig als Vermittler/innen fungieren (ebd., p. 29). Wer beispielsweise in Österreich einen Pflichtschulabschlusskurs oder eine Lehre absolvieren möchte, benötigt jedenfalls Grundkenntnisse in der Landessprache. Ehrenamtliche leisten also nicht nur direkte Integrationsarbeit, weil sie den Geflüchteten Deutsch beibringen. Indem sie ihnen dabei helfen weitere Bildungsangebote wahrnehmen zu können, tragen sie auch indirekt zur Integration bei (ebd.).

KLEIST (ebd., pp. 29 f.) weist jedoch auch auf professionelle Schwachstellen ehrenamtlicher Bildungsarbeit hin. Den Ehrenamtlichen sei häufig selbst bewusst, dass sie für den DaF/DaZ-Unterricht unzureichend ausgebildet sind. Selbst pensionierte Deutschlehrer/innen betonen, dass diese Art des Deutschunterrichts gänzlich anders funktioniert, als der Deutschunterricht für Muttersprachler/innen (Interview EHRENAMTLICHE UnTERSTÜTZERIN GemeInde A 2018). Dennoch war/ist ehrenamtlicher Deutschunterricht eine unverzichtbare Ressource der Integration Geflüchteter, nicht zuletzt deshalb, weil er soziale Beziehungen zwischen den Neuzugewanderten und den Mitgliedern der Aufnahmegesellschaft fördert. KLEIST plädiert daher dafür ,die professionellen Defizite des freiwilligen Bildungsangebots durch Fortbildung und Begleitung für Ehrenamtliche aufzufangen und deren Unterricht für Flüchtlinge zu verbessern, ohne den ehrenamtlichen und zivilgesellschaftlichen Charakter zu unterminieren“" (KLEIST 2017, p. 30). Hauptamt und Ehrenamt sollten als komplementär verstanden werden und nicht in Konkurrenz treten (ebd.). Auch wenn Verallgemeinerungen nicht zulässig sind, so kann bei professionellen Angeboten von einer größeren persönlichen Distanz zwischen Trainer/inne/n und Lernenden ausgegangen werden. Die berufliche Tätigkeit muss sich auf gewisse Zielvorgaben konzentrieren und ist zeitlich klar abgesteckt, während Ehrenamtliche häufig die nötige Zeit, Geduld, Empathie, aber auch das persönliche Interesse mitbringen, am Alltagsleben der Menschen teilzuhaben. Dies ermöglicht es den Freiwilligen, auf die spezifische Lebenssituation der Menschen einzugehen und zielgruppenadäquate Angebote für Geflüchtete umzusetzen.

\subsection{Zur Bedeutung von persönlichen Kontakten im Integrations- prozess}

HAN-BroICH zufolge umfasst Integration drei Dimensionen, wobei Ehrenamtliche in allen drei Bereichen einen bedeutenden Beitrag leisten können. Bei der emotional-seelischen Dimension geht es um die subjektiv empfundene Nähe beziehungsweise Distanz zur Aufnahmegesellschaft. Bei erfolgreicher Integration in diesem Bereich entwickeln die Zugewanderten positive Gefühle gegenüber ihrer Umwelt und der Aufnahmegesellschaft; sie fühlen sich als Mensch willkommen und akzeptiert. Bei der kognitiv-kulturellen Dimension steht das Erlernen der Sprache des Aufnahmelandes im Vordergrund. Parallel dazu findet eine „Angleichung in Wissen, Fertigkeiten [und] Normenkenntnisse[n]“" (HAN-BroICH 2012, p. 122). statt, die Menschen zu 
situationsadäquatem Verhalten befähigen. Grob zusammengefasst könnte man sagen, dass die kognitiv-kulturelle Dimension im Zeichen von Bildung und Lernen steht. Die sozial-strukturelle Dimension umfasst Freundschaften zu Mitgliedern der Aufnahmegesellschaft, Partizipation am gesellschaftlichen Leben sowie die Integration in den Wohnungs-, Bildungs- und Arbeitssektor. Am Beispiel des ehrenamtlichen Deutschunterrichts, welcher in allen Erhebungsgemeinden geleistet wurde, zeigt sich, wie die Dimensionen einander überlappen. Einerseits werden Sprachkenntnisse erworben, andererseits entstehen durch den intensiven Kontakt, der oft über den Kurs hinausgeht, enge Beziehungen, die das subjektive Wohlbefinden erhöhen können und der gesellschaftlichen Isolation von Asylwerber/innen entgegenwirken. Mit Blick auf die von HAN-BROICH definierten Dimensionen von Integration wird deutlich, dass der ehrenamtliche Sprachunterricht ein hohes integratives Potenzial hat.

„Der Deutschkurs ist natürlich eine Bildungsmaßnahme, aber [...] der ist durch und durch integrativ, ja. Es ist einfach ein soziales Netz, wo du mit allen Problemen und Sorgen hinkommen kannst und wo du alle Informationen bekommst und Hilfe und Unterstützung. Also es ist so richtige Basisarbeit ja. " (Interview EHRENAMTLICHE DeUTSCHLEHRERIN St. ANDRÄ-WÖrdERn 2018)

Sowohl HaN-Broich (2012, p. 157) als auch Simsa (2017, p. 84) sprechen in diesem Zusammenhang von „Beziehungsarbeit“, welche durch die ehrenamtlichen Tätigkeiten nebenbei stattfindet. Die Ehrenamtlichen würden insbesondere durch den Aufbau von Beziehungen im Bereich der seelisch-emotionalen Integration einen wesentlichen Beitrag leisten. Die Bedeutung von Ehrenamtlichen als „Gate-Keeper/ innen“ in die Aufnahmegesellschaft wird auch von LANGTHALER und TRAUnER (2009, p. 454) betont. Die freundliche Aufnahme in der Nachbarschaft, sowie ein generelles Wohlwollen Geflüchteten gegenüber, kann dabei entscheidend zum Sicherheitsgefühl und zum Wohlbefinden von Asylwerber/inne/n und Asylberechtigten beitragen. (vgl. AgER \& StRAng 2008, p. 180)

„Obwohl Ehrenamtliche nach ursprünglicher Aufgabenverteilung keine therapeutische bzw. psychosoziale Arbeit explizit zu leisten haben, sondern eher im kognitiv-kulturellen und sozial-strukturellen Bereich konkrete Hilfestellungen (z. B. Bildungs- und Betreuungsarbeit, Begegnung und praktische Lebenshilfe etc.) geben sollen, zeigt sich die größte Wirkung ihrer Arbeit gerade nicht in diesen die praktische Integration betreffenden Bereichen (z. B. im kognitiv-kulturellen und sozial-strukturellen Bereich), sondern vielmehr im seelisch-emotionalen Bereich. “ (HAN-BroICH 2012, p. 184)

Beziehungen sind jedoch keine eindimensionale Angelegenheit, sondern beruhen auf Gegenseitigkeit. Wenn Menschen flüchten, müssen sie oftmals auch ihre Familie und andere soziale Gefüge zurücklassen. Die Beziehungen zu den Ehrenamtlichen können im Laufe der Zeit die Qualität einer Ersatzbeziehung annehmen und die erfahrene Diskontinuität ein Stück weit ausgleichen; verloren gegangene soziale Bindungen können somit ergänzt oder neu hergestellt werden (HAN-BroICH 2012, p. 160). Manche Ehrenamt- 
liche bauen mit den Geflüchteten eine Eltern- oder Großelternersatzbeziehung auf. Die ehrenamtlichen Senior/inn/en-Lehrer/innen, die in der Gemeinde B mit den Kindern der örtlichen Volksschule und NMS spielerisch Deutsch lernen, übernehmen im Laufe der Zeit für manche Kinder eine Großelternrolle (vgl. LichtenwaGNER et al. 2019, p. 271).

Für viele Ehrenamtliche stellt der Kontakt mit den Geflüchteten eine Horizonterweiterung dar, was positiv hervorgehoben wird (vgl. Interview EHRENAMTLICHE Deutschlehrerin St. ANDRÄ-WÖRDERn 2018). Ähnliches wird auch aus Gemeinde A berichtet:

„Es war für uns persönlich auch gewissermaßen eine Bereicherung durch die Kontakte, die wir gehabt haben. Ein kultureller Austausch; also es ist gekocht worden. Wir haben dann auch so Begegnungstreffen gehabt in der Pfarre, im Pfarrsaal, alle paar Monate, wo musiziert worden ist, wo getanzt worden ist von den Frauen. Und wo sie [...] ihre selbst gefertigten Speisen [mitgebracht haben], also dass wir auch ihre Küche kennengelernt haben. Und das war eine ganz tolle Sache eigentlich. Es war nicht bloß eine Einbahnstraße, dass wir halt so viel Engagement gezeigt hätten, sondern es ist natürlich etwas auch zurückgekommen und das macht auch die Zufriedenheit aus, von denen, die dabei waren. “ (Interview Obmann „WillKommens-Verein“ Gemeinde A 2019)

Positive Beziehungsdynamiken können jedoch nicht nur zwischen den Geflüchteten und den Ehrenamtlichen entstehen, sondern werden mitunter auch zwischen den Freiwilligen wahrgenommen. Die Leiterin von ,St. Andrä-Wördern hilft“ erwähnte zum Beispiel, dass einige Unterstützer/innen untereinander neue Kontakte geknüpft und Freundschaften geschlossen haben (vgl. Interview LEITERIN „ST. ANDRÄ-WÖRDERN HILFT“ 2018).

Die Kontaktmöglichkeiten umfassen allerdings nicht nur den Besuch von Bildungsmaßnahmen und Interaktionen im Zuge von beruflichen oder ehrenamtlichen Tätigkeiten, sondern auch den Freizeitbereich. Wenn sich solche Gelegenheiten auf regelmäßiger Basis ergeben, kann dies mehrere positive Auswirkungen haben. Erstens ist „die persönliche Begegnung, wie man weiß, der Schlüssel zum Verständnis von anderen Menschen“ (Interview ObMann Verein „Grenzenlos“ 2018). Für die Geflüchteten ergibt sich dadurch auch die Möglichkeit, in den Deutschkursen erworbene Sprachkenntnisse anzuwenden, was wiederum gut aufgenommen wird, wenn andere Gemeindebewohner/innen merken, dass man sich mit den Asylwerber/inne/n und Asylberechtigten mit der Zeit recht gut unterhalten kann (vgl. Interview LEITERIN „ST. ANDRÄ-WÖRDERN HILFT“ 2018). Dadurch haben Geflüchtete auch die Möglichkeit selbstständig ihre Einkäufe zu erledigen und ihren Alltag zu gestalten. Darüber hinaus gibt es Anknüpfungspunkte, um ein soziales Netz aufzubauen, das über die Kontakte zu den Ehrenamtlichen hinausgeht.

Obwohl die zivilgesellschaftlichen Aktivitäten in den untersuchten Gemeinden von den Freiwilligen als erfolgreich und persönlich befriedigend wahrgenommen wurden, machte sich bei den ehrenamtlichen Unterstützer/inne/n immer wieder Ernüchterung 
breit. Zum Beispiel, wenn trotz aller Bemühungen gut integrierte Asylwerber/innen abgeschoben werden, Geflüchtete die Orte ohne Verabschiedung plötzlich verlassen oder wegen der Schließung von Quartieren aufgebaute Beziehungen nicht mehr fortgeführt werden können (vgl. Interview Ehrenamtliche Deutschlehrerin St. AndräWördern 2018; Interview Obmann „Willkommens-Verein“ Gemeinde A 2019). Das Engagement für Geflüchtete in den hier beschriebenen Formen lebt somit von der Unmittelbarkeit des persönlichen Kontaktes zwischen Ehrenamtlichen und Asylwerber/ inne/n beziehungsweise Asylberechtigten. Auch wenn die allgemeine Position von Geflüchteten in der österreichischen Gesellschaft immer wieder beklagt wird, bezieht sich der Großteil der Aktivitäten auf konkrete Personen, die auch lokal in den jeweiligen Gemeinden verortet sind.

\subsection{Der zeitliche und räumliche Faktor für den Integrationsprozess von Geflüchteten}

Für den Prozess der lokalen Integration von Asylwerber/inne/n und Asylberechtigten spielen die zeitlichen und räumlichen Gegebenheiten eine wichtige Rolle. Was den Faktor Zeit betrifft, verändern sich auch die Bedürfnisse an Unterstützung von Geflüchteten und damit die Angebote von Ehrenamtlichen in diesem Bereich. Der räumliche Faktor beschreibt in diesem Zusammenhang vor allem die gegebenen Möglichkeiten für Geflüchtete, die an einem bestimmten Ort wohnen und bezieht sich hauptsächlich auf Wohnraum, Arbeitsmöglichkeiten sowie Bildungsangebote und Mobilität.

\subsubsection{Der zeitliche Faktor im Integrationsprozess}

Simsa (2017, pp. 84 ff.) unterscheidet in Bezug auf zivilgesellschaftliche Unterstützung für Geflüchtete drei Phasen des „Life Cycle of Refugee Integration“: Die erste Phase des Ankommens (zum Beispiel 2015 und 2016 an den Bahnhöfen) ist von Nothilfe gekennzeichnet; es geht um die Organisation und Verteilung existentieller Güter wie Nahrung, Kleidung und Hygieneartikel. Die zweite Phase, die Zeit während des Asylverfahrens in der Grundversorgung, ist von Warten geprägt. In diesem Zeitraum spielt das freiwillige Deutschkursangebot durch Ehrenamtliche eine bedeutende Rolle. Die dritte und letzte Phase ist die Zeit nach Erhalt eines positiven Asylbescheids. Damit besteht sodann rechtlicher Anspruch auf den Besuch von Deutschkursen, weshalb andere Themen wie beispielsweise das Finden einer passenden Wohnung, eines Arbeitsplatzes beziehungsweise das generelle Zurechtfinden im Umgang mit Behörden in den Vordergrund treten.

In allen drei Abschnitten spielt ehrenamtliches Engagement eine wichtige Rolle, allerdings auf unterschiedliche Art und Weise. Obwohl es in St. Andrä-Wördern und in den Gemeinden im Mostviertel keine Notquartiere gab, wurden Neuankömmlinge zu Beginn vor allem mit Sachspenden unterstützt. Zur Organisation und Verteilung dieser 
Gegenstände wurden zentrale Ausgabestellen eingerichtet, wo die Hilfsgüter verteilt werden konnten. In dieser Phase wurde in beiden Erhebungskontexten eine erhöhte Bereitschaft der Zivilgesellschaft wahrgenommen sich einzubringen und sich in diesem Bereich zu engagieren. Mit dem danach relativ rasch wieder sinkenden Bedarf wurden diese Aktivitäten früher oder später jedoch wieder eingestellt.

Zeitgleich gab es allerdings in St. Andrä-Wördern und den Gemeinden des Mostviertels bereits das Angebot eines Deutschkurses, der von Freiwilligen organisiert wurde, und wenig später in St. Andrä-Wördern die Möglichkeit für Geflüchtete, für die Gemeinde gemeinnützige Tätigkeiten zu verrichten. ${ }^{29}$ Durch den „Grenzenlos“Solidarfonds wurde einigen Asylwerber/inne/n eine gewisse eigenständige Mobilität ermöglicht und der Zugang zu Bildungsmaßnahmen durch persönliche Kontakte der Ehrenamtlichen erschlossen. Diese Aktivitäten sind insofern wesentlich, da Asylwerber/ innen während der Dauer des Verfahrens vom regulären Arbeitsmarkt ausgeschlossen sind und dementsprechend nur über geringe finanzielle Mittel aus der Grundversorgung verfügen. Damit sind Beschäftigungsmöglichkeiten häufig eingeschränkt und das Bedürfnis einer geregelten Tagesstruktur meist hoch. Die erzwungene Untätigkeit während des Verfahrens wird oft als sehr zermürbend erlebt. Dieser Zeitraum zwischen der Asylantragstellung und dem Abschluss des Asylverfahrens wird sowohl in der Literatur als auch von den Geflüchteten und den Unterstützer/inne/n immer wieder als langwieriger Prozess des Wartens beschrieben. So bezeichnen ScHMIDINGER und GysI (2008) diese Periode als eine „lange Weile Langeweile“. Kontakte zu ehrenamtlichen Unterstützer/inne/n stellen hier besonders auf emotionaler Ebene eine wichtige Hilfestellung dar.

Wenn Geflüchtete einen Aufenthaltstitel in Österreich erhalten, ändern sich die rechtlichen Rahmenbedingungen maßgeblich. Innerhalb von vier Monaten muss das Grundversorgungsquartier verlassen werden und die Asylberechtigten haben Anspruch auf Sozialleistungen wie die Mindestsicherung sowie vollen Zugang zum Arbeitsmarkt (vgl. Interview LeITER BACH BILdungszentrum 2018). Durch die zusätzlichen finanziellen Mittel werden der Besuch eines Deutschkurses sowie die individuelle Mobilität der Asylberechtigten (oder subsidiär Schutzberechtigten) erleichtert. Allerdings müssen viele Bereiche des täglichen Lebens fortan selbst organisiert werden. So eröffnet sich erstmals die Möglichkeit, das Bundesland zu wechseln, sodass viele Geflüchtete, die während des Asylverfahrens in St. Andrä-Wördern untergebracht waren, beschließen nach Wien zu ziehen. Das trifft auch auf den allergrößten Teil der Asylberechtigten $\mathrm{zu}$, die in den Gemeinden im Mostviertel untergebracht waren. Auf die Gründe dafür wird im nächsten Abschnitt noch genauer eingegangen. Für diejenigen, welche in der Gemeinde bleiben wollen, ist es jedoch sehr schwierig eine Wohnung zu finden, da in St. Andrä-Wördern günstiger Wohnraum rar ist. In diesem Bereich können die Kontakte zu Ehrenamtlichen weiterhin eine große Bedeutung haben. Ebenso sind die Arbeitsmöglichkeiten in der näheren Umgebung für Asylberechtigte eingeschränkt, wodurch entsprechende persönliche Kontakte und Unterstützung im Bewerbungsprozess von

$29 \quad$ Für Details dazu siehe Abschnitt 7.6. 
besonderer Wichtigkeit sind. Es zeigt sich somit, dass sich ehrenamtliche Unterstützung für Geflüchtete zwar im Lauf der Zeit wandelt, aber dennoch auch später sehr bedeutsam ist.

Für die lokale Integration von Geflüchteten ist der Beitrag von Ehrenamtlichen ein ganz entscheidender. Simsa (2017, p. 84) unterscheidet im Zusammenhang mit diesen zivilgesellschaftlichen Aktivitäten in Bezug auf deren Intention zwischen,,initial treatment“ und ,integration measures“. „Initial treatment“ meint dabei vor allem die Soforthilfe für Geflüchtete unmittelbar nach deren Ankunft in Österreich und ist vorwiegend in der ersten Phase des „Life Cycle of Refugee Integration“ dominant. Hier spielt die humanitäre Motivation zur Hilfe für Geflüchtete aufgrund deren Bedürftigkeit eine große Rolle (vgl. FrIESE 2019). „Integration measures“, welche vor allem die zweite und dritte Phase betreffen, können vielfach unter dem häufig gebrauchten Schlagwort der Hilfe zur Selbsthilfe zusammengefasst werden. Auf den ersten Blick wirkt diese Unterscheidung wie eine klare Abgrenzung in der Charakteristik der beschriebenen Aktivitäten. Allerdings stellt der Vorgang des „Fußfassens“ in der Gesellschaft in Österreich keinen linearen Prozess dar. Es gibt demnach auch keinen geradlinigen Fortschritt in eine Richtung, sondern eine Gleichzeitigkeit von Ankommen und Integration. Dieser Zusammenhang wird von KoHLBACHER und Six-HoHENBALKen (2019, p. 8) folgendermaßen beschrieben: „Lineare Konzepte der strukturellen Integration stehen somit den zirkulären, individuellen (wie auch kollektiven) Fluchterfahrungen gegenüber und bedingen auf unterschiedlichen Ebenen Hürden, Rückschläge und besondere Herausforderungen." Somit kann weder das ,physische Ankommen“ noch die ,rechtliche Aufnahme“ in Österreich als endgültiger Abschluss angesehen werden (ebd.). Schließlich spielen Zuschreibungen aus der Aufnahmegesellschaft hier ebenfalls eine wichtige Rolle. So erfahren Geflüchtete häufig eine Einengung und Fortschreibung ihrer Identität auf den Flüchtlingsstatus (vgl. Interview EHRENAMTLICHE DeUTSCHLEHRERIN ST. ANDRÄ-WÖRDERN 2018). Zusätzlich betreffen Erfahrungen von struktureller und persönlicher Diskriminierung Geflüchtete oft noch viele Jahre nach ihrer Ankunft in Österreich. Damit sei an dieser Stelle auf die gesellschaftlichen Rahmenbedingungen verwiesen, die einen erheblichen Einfluss darauf haben, welche Teilhabemöglichkeiten und Verwirklichungschancen Geflüchteten offenstehen.

\subsubsection{Der räumliche Faktor im Integrationsprozess}

Die zentrale Bedeutung räumlicher Gegebenheiten unterscheidet sich ebenfalls je nachdem, ob Geflüchtete sich noch im Asylverfahren befinden oder dieses bereits positiv abgeschlossen haben. Besonders wichtig für die lokale Integration von Asylwerber/ inne/n ist die Lage der Grundversorgungsquartiere sowie deren öffentliche Verkehrsanbindung und für Asylberechtigte das Vorhandensein von geeignetem Wohnraum, Arbeitsmöglichkeiten und Bildungsangeboten in der Region.

In vielen Fällen befinden sich die Unterkünfte für Asylwerber/innen an abgelegenen Orten, eine Anbindung an öffentliche Verkehrsmittel ist dadurch häufig nur sehr 
eingeschränkt gegeben. Damit können Kursangebote oft nicht wahrgenommen werden, außer sie werden direkt in der Unterkunft oder in deren unmittelbarer Umgebung angeboten. Selbst wenn diese infrastrukturellen Möglichkeiten gegeben sind, stellt sich immer noch die Frage der Finanzierung von öffentlichen Verkehrsmitteln, um eine regelmäßige Kursteilnahme sicherzustellen. Viele Anbieter von Bildungsangeboten reagieren darauf, indem Fahrkarten für Geflüchtete bezahlt werden, auch wenn die finanzielle Belastung für die Organisationen dadurch recht hoch ist. Andernfalls wäre es jedoch oft schlicht nicht möglich, dass die Kurse überhaupt besucht werden können (vgl. Interview MitaRbeITERIN MigRATIONSABTEILUNG UND TRAINERIN BASISBILDUNG RoteS Kreuz Tulln 2018). Eine weitere Möglichkeit wäre eine Übernahme von Fahrtkosten für einzelne Geflüchtete durch andere Vereine wie etwa den „Grenzenlos“-Solidarfonds in St. Andrä-Wördern oder Privatpersonen. Der Nachteil ist dann jedoch, dass jenen Asylwerber/inne/n, die nicht über derartige Kontakte verfügen, die Teilnahme verwehrt bleibt. Eine weitere interessante Möglichkeit, insbesondere für den ländlichen Raum, ist die dezentrale Organisation von Bildungsmaßnahmen, wobei die Trainer/innen in die Gemeinde kommen (vgl. Abschnitt 6.1.1), um den Anfahrtsweg der Teilnehmer/ innen und die damit verbundenen Kosten gering zu halten (vgl. Interview SozIALARBeITERIN DiAKonie - mobiLer FlüChtlingSdienst 2018). Auf diese Weise können Fahrtkosten eingespart werden, allerdings eröffnen Monatskarten für Geflüchtete, um an Sprachkursen oder anderen Angeboten teilzunehmen, auch individuelle Mobilität für private Aktivitäten, was in diesem Fall wegfällt. Es zeigt sich somit recht deutlich, dass zusätzlich zu den rechtlichen Rahmenbedingungen und persönlichen Faktoren die spezifischen Möglichkeiten an den Aufenthaltsorten von Asylwerber/inne/n und Asylberechtigten relevant sind. Nach RYAN (2018) können diese als „Place Specific Opportunity Structures" bezeichnet werden.

Dem Vorhandensein entsprechender Perspektiven kann gerade bei der Wohnortentscheidung nach dem positiven Abschluss des Asylverfahrens eine hohe Bedeutung beigemessen werden. Aber auch hier spielt die Beziehung zu zivilgesellschaftlichen Unterstützer/inne/n eine wichtige Rolle. Einerseits können Geflüchtete von den vorhandenen Kontakten der Ehrenamtlichen am Wohnungs- und Arbeitsmarkt sowie im Bildungssektor profitieren und andererseits sind die entstandenen Freundschaften möglicherweise auch ein Grund, um nach dem positiven Abschluss des Verfahrens weiterhin in der Gemeinde zu wohnen. Dies setzt jedoch zufriedenstellende Partizipationsmöglichkeiten in wichtigen gesellschaftlichen Bereichen voraus.

Viele der Geflüchteten in St. Andrä-Wördern zieht es nach dem positiven Abschluss des Asylverfahrens nach Wien. Dafür werden unterschiedliche Gründe genannt, zum Beispiel die höheren Sozialleistungen in der Bundeshauptstadt (Interview KoOrdinatorin ehrenamtlicher DeutschKurs St. Andrä-Wördern 2018) oder vielfältigere Möglichkeiten auf dem Arbeits- und Wohnungsmarkt (Interview GEMEINDERAT FÜr Sozial- Gesundheitswesen: Integration, Migration, Freizeit, VeranSTALTUNGEN 2018). Schließlich gibt es häufig sehr vielfältige persönliche Gründe, warum jemand doch den städtischen Raum als Ort des eigenen Lebensmittelpunktes 
wählt. Für Geflüchtete können beispielsweise das Bedürfnis nach Kontaktmöglichkeiten zur eigenen ethnischen Community im urbanen Raum, die Verfügbarkeit von halal-Produkten oder Gebetsstätten in größeren Städten Gründe für den Ortswechsel darstellen (vgl. MeHL 2017, p. 128; INBAS-SozIALFORSCHUNG 2018, pp. 59 f.). Es gibt allerdings auch Aspekte, die nach dem positiven Abschluss des Asylverfahrens für einen Verbleib im ländlichen Raum sprechen. So werden die schöne Umgebung in St. Andrä-Wördern und der gute Umgang mit den Menschen, insbesondere in der Nachbarschaft, von den Geflüchteten immer wieder hervorgehoben (Interview AsYLWERBER IRAN 2018). Dadurch, dass die Klassen in den Schulen außerhalb Wiens kleiner sind und weniger andere Araber/innen dort unterrichtet werden, wird nach Ansicht eines syrischen Familienvaters die Integration seiner Söhne und Töchter im schulischen Kontext erleichtert (Interview AsYLBERECHTIGTER SYRIEN 2018). Zu all diesen Faktoren kommen noch die eigenen Präferenzen, lieber in der Stadt oder im ruralen Raum leben zu wollen. Einzelne Studien aus Deutschland belegen außerdem, dass ländliche Orte, von denen die nächste größere Stadt mit öffentlichen Verkehrsmitteln binnen weniger als einer Stunde erreicht werden konnte, für Geflüchtete tatsächlich eine Alternative zum Leben in der Großstadt darstellen, da somit Beziehungen zur eigenen ethnischen Community gepflegt werden können (INBAS-SozIALFORSCHUNG 2018, p. 59).

Die Verwirklichungschancen von Geflüchteten werden schon während des Asylverfahrens von den „Place Specific Opportunity Structures“ beeinflusst. So sind in St. Andrä-Wördern die Unterkünfte für Geflüchtete nicht isoliert in abgelegener Lage verortet, sondern in relativer Nähe zu einer öffentlichen Verkehrsanbindung. Mit der Unterstützung durch den „Grenzenlos“-Solidarfonds sind für einige dann auch die nötigen finanziellen Mittel vorhanden, diese Verkehrsverbindungen zu nutzen, was erheblich zu einer selbstbestimmten Alltagsgestaltung beiträgt. Der Kontakt zu den Ehrenamtlichen und der Deutschkurs wirken sich ebenfalls positiv auf die Verwirklichungschancen der Geflüchteten aus, da mit der einfachen Erreichbarkeit der Sprachkurse viele wichtige Kenntnisse vermittelt werden können, um sich besser auf eigene Faust zurechtzufinden.

In den Mostviertler Gemeinden hat sich unabhängig von der Intensität des ehrenamtlichen Engagements vor Ort gezeigt, dass der periphere (Gemeinden A und B), aber auch der zentrale ländliche Raum (Gemeinde C) durch das Quotensystem zur Lastenteilung kein dauerhafter Wohnort für die Geflüchteten wird, obwohl diese vor Ort teilweise sehr gute Beziehungen zur Gemeindebevölkerung aufgebaut haben. Diese Erfahrungen wirken sich auch nachhaltig auf das Engagement der Ehrenamtlichen aus. In der Gemeinde B bestanden zum Erhebungszeitpunkt im Jahr 2018 zwei Grundversorgungsquartiere, die bereits seit den 1980er Jahren betrieben wurden. Breites zivilgesellschaftliches Engagement, wie dies spontan in der Nachbargemeinde A im Herbst 2015 entstanden ist, gab es dort nicht. Der Direktor der NMS schilderte die Situation in der Gemeinde folgendermaßen:

„In den ersten Jahren [...] da haben die [Leute aus Gemeinde B] geholfen mit allem Möglichen. [...] Wenn das seit 1980 ist, jetzt haben wir 2018 [...]. [Es] 
machen nicht mehr alle mit. Es hat niemand mehr etwas gegen diese Leute, aber aktiv ihnen zulaufen [tun sie ihnen] auch nicht. Wenn es notwendig ist, okay, dann wird sicher etwas gemacht, aber es gibt keine großen Hilfsaktionen mehr. [...] Diese Geschichte haben wir hinter uns. "

Das Zitat zeigt, dass die Bevölkerung anfangs in den 1980er Jahren durchaus aufgeschlossen war, sich zivilgesellschaftlich für die Geflüchteten zu engagieren. Allerdings haben sie über Jahrzehnte die Erfahrung gemacht, dass sich diese Menschen nicht ansiedeln und zu bleibenden Nachbar/inne/n werden. Immer wieder kamen neue Geflüchtete in den Ort, die jedoch nur einige Monate, vielleicht ein Jahr, wohnen blieben, ehe sie die Gemeinde wieder verließen. Das hat auch Auswirkungen auf die langfristige Motivation von Ehrenamtlichen sich in diesem Bereich zu engagieren. Im Gegensatz dazu berichten mehrere Unterstützer/innen aus St. Andrä-Wördern, dass sie ihre Aktivitäten weniger als Hilfstätigkeiten an den Geflüchteten verstehen, sondern eher einen Raum schaffen möchten, in welchem interessierte Gemeindebewohner/innen zusammenkommen, sich austauschen und eine gute Zeit haben können.

\subsection{Zur lokalen Einbettung des ehrenamtlichen Engagements}

Um dieses Kapitel abzuschließen soll hier noch auf wichtige Schlüsselpersonen und -institutionen eingegangen werden, deren Aktivitäten Integration maßgeblich mitgestalten können. Relevant ist hierbei auch die Beziehung zwischen den unterschiedlichen Akteur/inn/en. Es zeigt sich, dass ein gemeinsames Verständnis für die Wichtigkeit von lokaler Integration der Geflüchteten einen bedeutenden Beitrag zum Gelingen dieses Prozesses leisten kann.

\subsubsection{Zur Rolle der Pfarren und Bürgermeister im Mostviertel}

Für das freiwillige Engagement in den Gemeinden spielen die Pfarren und der/die Bürgermeister/in eine wesentliche Rolle. Sie verleihen dem Engagement im Idealfall den nötigen moralischen oder politischen Rückhalt. EvERS und KLIE heben beispielsweise die moralische Autorität von Kirchen hervor, die es in der Regel vermögen, Menschen unterschiedlicher sozialer und kultureller Herkunft zusammenzubringen. Sie verweisen in diesem Zusammenhang explizit auf die Sonderrolle von Kirchen im Kontext ehrenamtlichen Engagements für Geflüchtete (EvERS \& KLIE 2018, pp. 528 f.).

Sowohl in der Gemeinde A als auch in der Gemeinde $\mathrm{C}$ war das freiwillige Engagement - wenn auch zu unterschiedlichem Grad - in die Strukturen der örtlichen Pfarre eingebettet. In der Gemeinde A war die Vereinsgründung ursächlich mit der Pfarre beziehungsweise mit bestimmten Personen, die sich bereits jahrelang über die Pfarre für soziale Angelegenheiten engagierten, verbunden.

„Ja, es hat bei uns in der Pfarre immer schon ein Engagement gegeben. Es hat ja mehrere Fluchtbewegungen gegeben. [...] Und da gibt es eigentlich eine 
Tradition, auch eine pfarrliche Tradition, also dass Flüchtlinge immer unterstützt worden sind. “ (OBMANn „Willkommens-Verein“ GEMEINDE A 2019)

Beim rezenten Engagement war die Pfarre unter anderem durch die Organisation von kulinarischen Begegnungstreffen im Pfarrsaal involviert, über den Pfarrflohmarkt wurden Sachspenden verteilt und Geflüchtete erhielten zum Beispiel die Möglichkeit im Kirchenchor mitzusingen (Interview Obmann „WiLlkommens-Verein“ Gemeinde A 2019). Darüber hinaus diente die Pfarrzeitung als Medium zur Mobilisierung von Freiwilligen (Interview EhrenamtLiche UnterstütZERIN GemeInde A 2018).

Neben der Kirche spielt auch der beziehungsweise die Bürgermeister/in eine wesentliche Rolle. Er oder sie kann das Engagement zum Beispiel wohlwollend mittragen und den Aktivitäten politischen Rückhalt bieten. Ist dies der Fall, wirken sich die Loyalität oder Unterstützung für Engagierte durch dieses politische Amt positiv auf die Motivation der Freiwilligen aus (LIDZBA 2018, pp. 280 f.). Das Amt des Bürgermeisters verlangt es jedoch, den Großteil der Bevölkerung zu vertreten und von Aktionen mit Provokationspotential Abstand zu nehmen.

Für die Gemeinde A wurde die allgemeine Stimmung bezüglich der Aufnahme von Geflüchteten im Herbst 2015 als ,zweigeteilt“ (Interview OBMANN „WillKommensVerein“ Gemeinde A 2019) beschrieben. Trotz großer Hilfsbereitschaft gab es auch eine Gruppe von Menschen, die dem Engagement für die Asylwerber/innen reserviert gegenüberstand. Dem Bürgermeister fiel die herausfordernde Aufgabe zu, im Rahmen der gesetzlichen Vorgaben zu agieren und Unruhen in der Bevölkerung zu vermeiden. Als Beispiel wurde unabhängig voneinander sowohl vom Bürgermeister als auch vom Obmann des „Willkommens-Vereins“ folgender Vorfall geschildert: Die Gemeinde verfügt über ein Freibad, dessen Badeordnung für Frauen das Tragen eines landesüblichen Badeanzugs oder Bikinis vorsieht. Während sich der Obmann des ,WillkommensVereins“ sowie einige Unterstützer/innen für die Erlaubnis mit Burkini baden gehen zu dürfen aussprachen, lehnte ein Großteil der Gemeindebevölkerung eine Änderung der lokalen Badeordnung ab. Der Bürgermeister fasste die Stimmungslage mit folgenden Worten zusammen: „Das war ein Diskussionsthema an allen Wirtshaustischen - sehr emotional“". Die Gemeindeleitung entschied daraufhin, die Badeordnung nicht zu ändern, woraufhin sich die aufgeregte Stimmung bei der Mehrheit der Gemeindebevölkerung wieder beruhigte (Interview OBMANN ,WILLKommens-VereIN“ GeMEINDE A 2019; Interview BÜrgERMEISTER GEMEINDE A 2018).

Im Fall von der Gemeinde A wurde das Agieren des Bürgermeisters von Seiten des Obmanns des „Willkommens-Vereins“ als „vorsichtig taktierend“ beschrieben. Der Bürgermeister war nämlich zeitweise sogar selbst in den Verein involviert, musste jedoch vorsichtig ausloten, welche Zugeständnisse an die Geflüchteten innerhalb der Gemeindebevölkerung keine größere Empörung nach sich ziehen würden. Dieses Beispiel zeigt, dass zivilgesellschaftliches Engagement für Geflüchtete - anders als das klassische Ehrenamt, welches in gesellschaftlich weniger kontroversen Bereichen wie Sport, Jugendarbeit oder Gesundheit aktiv ist - meist eine stärkere polarisierende 
Komponente aufweist. KaraKaYali (2017, p. 19) verweist in diesem Zusammenhang darauf, dass sich die Ehrenamtlichen oftmals vor Nachbar/inne/n, Kolleg/inn/en oder Verwandten rechtfertigen müssten. Dies traf in der Gemeinde A auch auf den Bürgermeister zu.

Ein weiteres Beispiel betrifft die Möglichkeit der gering entlohnten gemeinnützigen Tätigkeit für Geflüchtete, welche ebenfalls ein heikles Thema für die politische Gemeindeleitung darstellte. Einerseits bestünde darin eine Chance, den Asylwerber/ inne/n während der Wartezeit im Verfahren eine sinnvolle Beschäftigung zu für die Gemeinde kostengünstigen Bedingungen anzubieten. Andererseits wurde von zwei Interviewpartnern thematisiert, dass die Bürgermeister von einem Teil der arbeitslosen Gemeindebevölkerung für dieses Vorgehen kritisiert wurden, mit dem Argument, die Geflüchteten, würden ihnen die Arbeitsplätze wegnehmen (Interview OBMANN „Willkommens-Verein“ Gemeinde A 2019; Interview Amtsleiter Gemeinde C 2018).

Lokale Pfarren vermögen es häufig, Raum für Begegnung bereitzustellen und ehrenamtliches Engagement für Geflüchtete in moralische Werte einzubetten. Doch auch gemeindepolitische Akteur/inn/e/n, wie zum Beispiel Bürgermeister/innen und Amtsleiter/innen, gestalten die Rahmenbedingungen für die lokale Integration maßgeblich mit. Kirchen und Gemeindepolitiker/innen können dem ehrenamtlichen Engagement also den nötigen moralischen und politischen Rückhalt geben und eine proaktive Auseinandersetzung auf diesem Themengebiet fördern.

\subsubsection{Die Rollenverteilung auf Gemeindeebene in St. Andrä-Wördern}

In St. Andrä-Wördern gibt es im Gemeinderat innerhalb des Sozial- und Kulturausschusses auch einen Schwerpunkt zu Migration und Integration und einen dafür zuständigen Gemeinderat, der im Jahr 2018 von der SPÖ gestellt wurde. Als konkrete gemeindeeigene Integrationsmaßnahmen sind sowohl die „Integrationsgespräche“ als auch die Arbeitsmöglichkeit für Asylwerber ${ }^{30}$ im Bauhof zu nennen. In den Jahren 2013 bis 2017 fanden in der Gemeinde in Kooperation mit dem Projekt „ZusammenReden“ der Caritas Wien mindestens zweimal jährlich sogenannte „Integrationsgespräche“ statt. Dabei wurden integrationsrelevante Themen mit lokalen und externen Expert/inn/en unter Einbindung der Bevölkerung diskutiert. Die Ziele waren vorrangig, sich sachlich über diese Inhalte austauschen zu können, unterschiedliche Perspektiven aufzuzeigen und Informationen zu vermitteln. Darüber hinaus gibt es in Niederösterreich die Möglichkeit, dass Asylwerber/innen im Rahmen von gemeinnützigen Tätigkeiten im Gemeindedienst eingesetzt werden können. Auch in St. Andrä-Wördern wurde von dieser Regelung eine Zeit lang Gebrauch gemacht. Die zentralen Aufgabengebiete lagen dabei in den Sommermonaten in der Grünraumpflege, Mähtätigkeiten, Instandhaltungsarbeiten und ähnlichen Beschäftigungen. Der Stundenlohn betrug fünf Euro, was bei einer Zuverdienstgrenze von 110 Euro monatlich in der Grundversorgung die Einsatzdauer der Asylwerber stark einschränkte (vgl. Interview LeITER BAUHOF ST. ANDRÄ-

30 An diesem Programm beteiligten sich nur männliche Asylwerber. 
WÖRDERn 2018). Trotzdem waren die positiven Auswirkungen dieses Programms auf mehreren Ebenen spürbar. Einerseits profitiert der Bauhof durch die zusätzlichen günstigen Arbeitskräfte, andererseits haben auch die Asylwerber viele Vorteile durch dieses Projekt: Sie können sich nicht nur eigenes Geld verdienen, sondern zumindest an einigen Tagen einer geordneten und sinnvollen Aufgabe nachgehen (vgl. Interview LEITER „GRENZENLOS“-SOLIDARFONDS 2018). Darüber hinaus werden die Geflüchteten in der Gemeinde verstärkt wahrgenommen und die Bevölkerung merkt, dass sie gewillt sind, etwas beizutragen (vgl. Interview OBMANn Verein „Grenzenlos“ 2018). ${ }^{31}$ Es gibt dazu allerdings nicht nur in der Gemeinde $\mathrm{C}$ kritische Stimmen. Eine ehrenamtliche Unterstützerin in St. Andrä-Wördern bemängelte beispielsweise die geringe Entlohnung, die Asylwerber/innen für ihre Tätigkeiten erhalten.

Zusätzlich zu diesen eigenen Aktivitäten versucht die Gemeinde Angebote von Ehrenamtlichen zu unterstützen. So werden für den Deutschkurs Räumlichkeiten und Kopiermöglichkeiten zur Verfügung gestellt, der Verein „Grenzenlos“ finanziell gefördert und auch die Kosten für die Nachmittagsbetreuungsplätze für Kinder von geflüchteten Familien übernommen. Ebenso wirkt sich die explizite Zuständigkeit eines Gemeinderates für diese Themen positiv aus, da es eine konkrete Ansprechperson gibt, die eine koordinierende Funktion ausübt (vgl. Interview Mitarbeiterin Projekt Missing Link/ZusammenReden Der Caritas Wien 2018).

Ein Großteil der operativen Integrationsarbeit, wie beispielsweise der Deutschkurs, unterschiedliche Veranstaltungen und persönliche Unterstützung für einzelne Geflüchtete, wird in St. Andrä-Wördern allerdings von Ehrenamtlichen geleistet. Die Unterstützung dieser Maßnahmen durch die Gemeinde findet eher auf infrastruktureller Ebene statt. Daraus ergibt sich auch, dass die Freiwilligen in vielen Fällen mehr direkten Kontakt zu den Geflüchteten haben als Gemeindevertreter/innen. Außerdem beziehen sich die Aktivitäten auf unterschiedliche Dimensionen von Integration: Während die Arbeitsmöglichkeit für die Gemeinde vor allem zur sozial-strukturellen Integration von Asylwerber/inne/n einen wichtigen Beitrag leistet, sind die Angebote von „Grenzenlos““ und den beiden Initiativen vorwiegend in der emotional-seelischen und kognitiv-kulturellen Dimension wirksam. Es zeigt sich, dass die Bereiche, in welchen sich ehrenamtliches Engagement besonders positiv auswirkt, von offizieller Gemeindeseite nur schwer abgedeckt werden können. Andererseits verfügt die Zivilgesellschaft über keine Möglichkeiten in ähnlicher Form Beschäftigung für Geflüchtete anzubieten, die auch finanziell entlohnt wird. Somit kann eine unterschiedliche Rollenverteilung zwischen den Ehrenamtlichen und der Gemeinde festgestellt werden.

Dass die Gemeinde den Auftrag zur Integration der Geflüchteten zu einem großen Teil auf die Zivilgesellschaft überträgt, wird von den Ehrenamtlichen dabei nicht unbedingt gutgeheißen (vgl. Interview LEITERIN „ST. ANDRÄ-WÖRDERN HILFT“ 2018). Eine Ehrenamtliche betonte, dass die gelungene Integration vieler Geflüchteter in der Gemeinde vor allem auf das zivilgesellschaftliche Engagement in diesem Bereich zurück-

31 Die Möglichkeit der gemeinnützigen Arbeit für Asylwerber wurde mit der Schließung der Unterkünfte nicht mehr angeboten. 
zuführen ist: „Es funktioniert, weil es den Verein ,Grenzenlos` gibt“ (Interview EHRENAmTliche Deutschlehrerin St. AnDrä-Wördern 2018). Auch wenn sich ehrenamtliche Unterstützer/innen mehr direktes Engagement von Gemeindeseite wünschen, ist die indirekte Förderung der Zivilgesellschaft doch ein wesentliches Zeichen dafür, dass es auf Gemeindeebene wichtig ist, sich mit diesem Thema auseinanderzusetzen. So wurde mittels der Berichterstattung über die Aktivitäten der Initiativen und des Vereins „Grenzenlos“ in der Gemeindezeitung immer wieder entsprechende Öffentlichkeitsarbeit geleistet. Die Unterstützung seitens der Gemeinde stellt somit einen förderlichen Faktor für zivilgesellschaftliche Aktivitäten dar (vgl. Interview MitaRbeITERIN PROJEKT Missing Link/ZusammenReden Der Caritas Wien 2018).

Die Aktivitäten von Ehrenamtlichen stellen zwar den Ausgangspunkt zur Schaffung von günstigen Rahmenbedingungen für lokale Integration in St. Andrä-Wördern dar, aber auch von Gemeindeseite wird diesbezüglich einiges beigetragen. Dies umfasst einerseits den politischen Willen sich mit dem Thema Integration auseinanderzusetzen und andererseits die Unterstützung der zivilgesellschaftlichen Initiativen. Somit werden die Rahmenbedingungen zur lokalen Integration von Geflüchteten in der Gemeinde durch verschiedene Akteur/inn/e/n mitgestaltet. Für die Lebensrealität von Asylwerber/ inne/n und Asylberechtigen in Österreich allgemein, aber auch in St. Andrä-Wördern im Speziellen, sind zusammenfassend vor allem der Aufenthaltsstatus, die Gegebenheiten vor Ort, entsprechende Unterstützungsmaßnahmen und individuelle Faktoren von entscheidender Bedeutung. All diese Dinge verändern sich im Lauf der Zeit, sodass eine Analyse von Integrationsprozessen eine Verortung im zeitlichen und räumlichen Kontext zwingend vorzunehmen hat.

\subsection{Fazit}

Die Ergebnisse der empirischen Beispiele sowie die einschlägige Fachliteratur zeigen, dass ländliche Gemeinden sowie insbesondere die ehrenamtlich engagierte Bevölkerung bei der dringenden Bewältigung der Integrationsaufgabe seit 2015 einen wesentlichen Beitrag geleistet haben. Im Bereich der freiwilligen Unterstützung von Geflüchteten zeichnete sich in den letzten Jahren ein Strukturwandel des Ehrenamtes ab, das stärker zeitlich begrenzt ist und bei dem das inhaltliche Interesse an der konkreten Aufgabe deutlich im Vordergrund steht. Die neu gegründeten Initiativen organisieren sich selbst und weisen eher flache Hierarchien auf (SCHÜHRER 2019, pp. 13 ff.; BMFSFJ 2017, pp. 121 ff.). Obwohl sich die ,neuen Ehrenamtlichen“ hauptsächlich in Bereichen der praktischen Hilfe engagieren (zum Beispiel im Rahmen ehrenamtlicher Deutschkurse) und meist keine explizit politisch-transformativen Ziele verfolgen, sollte ihr Engagement dennoch nicht als unpolitisch verstanden werden (vgl. FLEISCHMANN \& SteinhilPer 2017; VANDEVoordt \& Verschraegen 2019; Karakayali 2017).

Die ehrenamtlichen Deutschkurse sind ein gutes Beispiel, um die Vielschichtigkeit zivilgesellschaftlichen Engagements sichtbar zu machen. Sie sind eine bedeut- 
same niederschwellige Bildungsmaßnahme, die als „Hilfe zur Selbsthilfe“ dient. Das integrative Potenzial ist jedoch nicht nur auf den Bereich der Sprachkenntnisse begrenzt. Durch den intensiven Kontakt, den Ehrenamtliche und Geflüchtete über den Deutschkurs aufbauen, entstehen mitunter freundschaftliche Beziehungen, die für beide Seiten als wertvoll empfunden werden. Ehrenamtliche haben eine Schlüsselrolle inne und fungieren gewissermaßen als „Gate-Keeper/innen“ zwischen den Geflüchteten und der Aufnahmegesellschaft (vgl. Kleist 2017, pp. 27 ff.; Han-Broich 2012, p. 157; LANGTHALER \& TRAUner 2009, p. 454).

Gerade im ländlichen Raum sind Geflüchtete besonders auf die Unterstützung von Ehrenamtlichen angewiesen, was vielerorts auch sehr gut funktionierte. Doch obwohl die Geflüchteten (durch die Hilfe einiger Ehrenamtlicher) häufig gute Kontakte zur lokalen Bevölkerung aufbauen, hat sich gezeigt, dass sie (periphere) ländliche Regionen, die geringere Verwirklichungschancen bieten, nicht als dauerhaften Wohnsitz in Betracht ziehen (für Deutschland vgl. INBAS SozIALFORSCHUnG 2018, pp. 58 ff.; MeHL 2017). Bei manchen Unterstützer/inne/n führt das im Laufe der Zeit auch zu Ernüchterung und sie ziehen sich aus der ehrenamtlichen Tätigkeit wieder zurück. Neben vielfältigen individuellen Gründen für die Wohnsitzwahl sollten auch (infra-) strukturelle Faktoren nicht außer Acht gelassen werden. Die Rolle des zivilgesellschaftlichen Engagements für die Ansiedelung Neuzugewanderter, also für deren langfristige lokale Integration, sollte somit nicht überschätzt werden. Ist die Bindung der Geflüchteten an den ländlichen Raum gewünscht, bedarf diese Aufgabe politisch-strategischer Planung auf verschiedenen Ebenen und in vielen Strukturbereichen. Dazu zählt beispielsweise, langfristig den Erwerb von Wohneigentum sowie individuelle Mobilität durch öffentlichen Personennahverkehr zu ermöglichen, dezentrale Bildungsangebote bereitzustellen und umfassende Konzepte zur Arbeitsmarktintegration zu entwickeln. Dies sind alles Themen, die die Bevölkerung der Aufnahmegesellschaft in ländlichen Gebieten gleichermaßen betreffen und von deren Reformierung sie ebenso profitieren könnte. Darüber hinaus müssen Kommunikations- und Kooperationsstrukturen in der Verwaltung geschaffen werden, die auch die Zivilgesellschaft beziehungsweise ehrenamtlich Engagierte sowie die Geflüchteten einbinden (vgl. OHLinger, Schweiger \& VeyHl 2017; Mehl 2017). 